\title{
ESTUDIOS CROMOSOMICOS EN ESPECIES ARGENTINAS DE VERNONIA (ASTERACEAE)
}

\author{
por MASSIMILIANO DEMATTEIS ${ }^{1}$
}

\section{Summary}

\begin{abstract}
The chromosomes of eleven Vernonia species from Argentina are studied. The results include counts in seven species that have never been investigated cytologically. The karyoiype of six taxa are analized, five of them are described ior the first time: $V$. chamaedrys $n=17,2 n=34=22 m$ $+12 \mathrm{sm} ; V$. loretensis $n=17,2 n=34=20 m+14 \mathrm{sm} ; V$. rubricaulis $n=16,2 n=32=20 m+12 \mathrm{sm}$; $V$. remotiflora $n=14,2 n=28=16 m+12 \mathrm{sm}$ and $V$. lepidifera $n=10,2 n=20=8 m+12 \mathrm{sm}$. The record of $2 n=4 x=28$ for $v$. remotiflora supports the existence of the basic chromosome number $x=7$. Previously reported chromosome counts show that Vernonia is multibasic with $x=7,9,10,11$, $12,15,17$ and $1 \mathrm{C}$. The significance of the results is discussed in relation to chromosomal data available for the genus.
\end{abstract}

\section{Introducción}

Ei género Vernonia Schreb. posee ca. 1.000 especies distribuidas en las regiones cálidas y templadas de Asiá, Africa y América. Para la República Argentina se har citado 46 especies que habitan en su mayoría en las provincias septentrionales, extendiéndose algunas hasta ei paraleio $38^{\circ}$.

Los estudios cromosómicos realizados hasta el momento en especies argentinas comprenden el recuento de 16 entidades (Núñez en Cabrera, 1944; Covas \& Hunziker, 1954; Hunter, 1964; Coleman, 1968; Jones, 1970, 1974, 1979 y 1982; Bernardello, 1986; Sundberg et al., 1986, Gaiiano \& Hunziker, 1987; Stutts, 1988; Hunziker et al., 1990; y ei análisis cariotípico de tres de ellas: $V$. flexuosa Sims, $V$ nudiflora Less. y $V$. polyphylla Sch.Bip. (Ruas et al., 1991).

En el presente trabajo se estudian cromosómicamente once especies pertenecientes a la sect. Lepidaploa (Cass.) DC. Se presenta ei primer recuento para $V$ echioides, $V$. lepidifera, $V$. loretensis, $V$. niederleinii, $V$. remotiflora, $V$. teyucuarensis y $V$. verbascifolia, y se analizan los

\footnotetext{
' Becario de la Secretaría General de Ciencia y Técnica de la UNNE. Instituto de Botánica del Nordeste, C.C. 209, 3400 Corrientes, Argentina.
}

cariotipos de seis especies, cinco de los cuales son descriptos por primera vez.

\section{Material y Métodos}

Los datos del material analizado se detallan en la Tabla 1. Para el estudio de los cromosomas meióticos se fijaron capítulos en aicohoi etílico absoluto: ácido láctico 5:1 (Fernández, 1973). Los preparados se realizaron a partir de anteras por apiastado y tinción en carmin acético al 3\%.

El análisis de los cromosomas mitóticos se realizó en ápices de raices. Estas fueron pretrataāas con 8-Oxiquinoleína 0,002 M durante 4 horas a temperatura ambiente y fijadas en aicohol etílico absoluto: ácido acético glacial (3:1). La coloración se realizó siguiendo la técnica de Feulgen.

Para la elaboración de los idiogramas, longitud total del cariotipo (LTC), índice centromérico medio (IC) y longitud cromosómica media (LM) se utilizaron los datos de 10 placas metafásicas.

La nomenclatura utilizada en la descripción de los cariotipos es la propuesta por Levan et al. (1964). La morfología de los cromosomas se determinó utilizando el índice centromérico (brazo corto x 100 / largo total del cromosoma). 
En las especies analizadas se distinguieron cromosomas metacéntricos $(m)=50-37,5$ y submetacéntricos $(\mathrm{sm})=37,5-25$.

\section{Resultados}

En la Tabla 1 se detallan los números cromosómicos obtenidos a partir del análisis mitótico y meiótico. Los datos del cariotipo de las seis especies estudiadas se consignan en la Tabla 2. A continuación se describen las características más destacadas de cada uno de los cariotipos.

$V$. chamaedrys, $2 \mathrm{n}=34$, el cariotipo está formado por $22 \mathrm{~m}+12 \mathrm{sm}$ (Fig. $1 \mathrm{~A}$ ). La longitud de sus cromosomas varía de 0,88 a 2,46 $\mu \mathrm{m}$. En el

Tabla 1. Material estudiado y resultados obtenidos.

\begin{tabular}{|c|c|c|c|c|}
\hline Taxón & $\mathrm{n}$ & $2 n$ & Testigos & Fig. \\
\hline \multicolumn{5}{|l|}{ Subsect. Chamaedrys } \\
\hline$V$. chamaedrys Less. & 17 & 34 & $\begin{array}{l}\text { Dematteis 316. Misiones, Dep. Capital, Posadas } \\
\text { (MNES, CTES, SI). }\end{array}$ & $2 \mathrm{~A}$ \\
\hline \multicolumn{5}{|l|}{ Subsect. Fchioides } \\
\hline V. echioides Less.* & 34 & - & $\begin{array}{l}\text { Dematteis et al. 283. Misiones, Dep. L. N. Alem, } \\
\text { Ruta } 14 \text { a } 15 \mathrm{~km} \text { de Cerro Azul (MNES, CTES, SI). }\end{array}$ & \\
\hline \multicolumn{5}{|l|}{ Subsect. Nudiflorae } \\
\hline V. loretensis Hieron.* & 17 & 34 & $\begin{array}{l}\text { Dematteis et al. 294. Misiones, Dep. San Ignacio, } \\
\text { San Ignacio (MNES, CTES, SI). }\end{array}$ & 2B \\
\hline \multicolumn{5}{|l|}{ Subsect. Remotiflorae } \\
\hline$V$ polyphylla Sch.Bip.** & - & 34 & $\begin{array}{l}\text { Dematteis et al. 295. Misiones, Dep. San Ignacio, } \\
\text { San Ignacio (MNES, CTES, SI). }\end{array}$ & $2 \mathrm{C}$ \\
\hline V. niederleinii Hieron.* & - & 90 & $\begin{array}{l}\text { Dematteis et al. } 480 \text {. Misiones, Dep. Cainguás, } \\
\text { Campo Grande (MNES). }\end{array}$ & \\
\hline V. teyucuarensis Cabrera* & 81 & 162 & $\begin{array}{l}\text { Dematteis 298. Misiones, Dep. San Ignacio, } \\
\text { camino a Teyú Cuaré (MNES, CTES, SI). }\end{array}$ & \\
\hline $\begin{array}{l}\text { V. rubricaulis Humb. } \\
\text { et Bonpi. ** }\end{array}$ & 16 & 32 & $\begin{array}{l}\text { Dematteis 284. Misiones, Dep. L. N. Alem, Ruta } 14 \text { a } \\
15 \text { km de Cerro Azul (MNES, CTES, SI). }\end{array}$ & $2 \mathrm{D}$ \\
\hline V. remotiflora L. C. Rich.* & 14 & 28 & $\begin{array}{l}\text { Dematteis 273. Misiones, Dep. San Javier, Cerro } \\
\text { El Monje (MNES, CTES). }\end{array}$ & $\begin{array}{l}2 \mathrm{E} \\
3 \mathrm{~A}\end{array}$ \\
\hline V. verbascifolia Less.* & 10 & - & $\begin{array}{l}\text { Dematteis 341. Misiones, Dep. Capital, Arroyo } \\
\text { Zaimán (MNES). }\end{array}$ & $3 B$ \\
\hline \multicolumn{5}{|l|}{ Subsect. Flexuosae } \\
\hline$V$. lepidifera Chodat* & 10 & 20 & $\begin{array}{l}\text { Dematteis 312. Misiones, Dep. Capital, Posadas } \\
\text { (MNES, CTES, SI). }\end{array}$ & $\begin{array}{l}2 \mathrm{~F} \\
3 \mathrm{D}\end{array}$ \\
\hline V. platensis (Spreng.) Less. & 20 & - & $\begin{array}{l}\text { Dematteis el al. } 442 . \text { Misiones, Dep. Capital, } \\
\text { Arroyo Zaimán (MNES, CTES, SI). }\end{array}$ & $3 C$ \\
\hline
\end{tabular}

* primer recuento para la especie

** número que difiere de recuentos anteriores 
Tabla 2. Características cromosómicas de las seis especies estudiadas.

\begin{tabular}{lccccc}
\hline Taxón & $\begin{array}{c}\text { Fórmula } \\
\text { cariotipica }\end{array}$ & $\begin{array}{c}\text { ITC - E.S.* } \\
(\mu \mathrm{m})\end{array}$ & $\begin{array}{c}\text { LM } \\
(\mu \mathrm{m})\end{array}$ & $\begin{array}{c}\text { Rango de } \\
\text { variación }(\mu \mathrm{m})\end{array}$ & TC - E.S.* \\
\hline V. chamaedrys & $22 \mathrm{~m}+12 \mathrm{sm}$ & $25,63 \pm 0,63$ & 1,50 & $0,88-2,46$ & $40,59 \pm 0,48$ \\
V. loretensis & $20 \mathrm{~m}+14 \mathrm{sm}$ & $21,15 \pm 0,54$ & 1,24 & $0,86-1,90$ & $41,67 \pm 0,49$ \\
V. polyphylla & $20 \mathrm{~m}+14 \mathrm{sm}$ & $25,54 \pm 0,58$ & 1,50 & $0,85-2,19$ & $40,60 \pm 0,46$ \\
$V$. rubricaulis & $20 \mathrm{~m}+12 \mathrm{sm}$ & $18,70 \pm 0,39$ & 1,16 & $0,79-1,55$ & $41,87 \pm 0,30$ \\
$V$. remotiflora & $16 \mathrm{~m}+12 \mathrm{sm}$ & $18,91 \pm 1,15$ & 1,35 & $1,15-2,03$ & $40,80 \pm 0,43$ \\
V. lepidifera & $8 \mathrm{~m}+12 \mathrm{sm}$ & $18,13 \pm 0,37$ & 1,81 & $1,08-2,75$ & $38,61 \pm 0,32$ \\
\hline
\end{tabular}

* error estándar

brazo largo de los pares $16 \mathrm{sm}$ y $17 \mathrm{sm}$ se pudo observar la presencia de satélite.

$V$. loretensis, $2 \mathrm{n}=34$, ia fórmula cariotípica está integrada por $20 \mathrm{~m}+14 \mathrm{sm}$ (Fig. 1B), observándose el satélite en el brazo largo del par $15 \mathrm{sm}$. El tamaño de sus cromosomas varía de 0,86 a $1,90 \mu \mathrm{m}$.

$V$. polyphylla, $2 \mathrm{n}=34$, el cariotipo está constituido por $20 \mathrm{~m}+14 \mathrm{sm}$ (Fig. 1C) y presenta un satélite en el brazo largo del par 17sm. La longitud de sus cromosomas varía de 0,85 a 2,19 $\mu \mathrm{m}$, con un promedio de $1,50 \mu \mathrm{m}$.

$V$. rubricaulis, $2 \mathrm{n}=32$, presenta el cariotipo formado por $20 \mathrm{~m}+12 \mathrm{sm}$ (Fig. 1D) y el satélite se halla en el brazo largo del par $1 \mathrm{~m}$. Posee los cromosomas más pequeños de las especies estudiadas, el promedio es de 1,16 $\mu \mathrm{m}$, oscilando entre 0,79 y $1,55 \mu \mathrm{m}$.

$V$. remotiflora, $2 \mathrm{n}=28$, muestra un cariotipo formado por $16 \mathrm{~m}+12 \mathrm{sm}$ (Fig. 1E), en el brazo corto del par $14 \mathrm{sm}$ presenta un satélite. El tamaño de los cromosomas varía de 1,15 a 2,03 $\mu \mathrm{m}$.

$V$. lepidifera, $2 \mathrm{n}=20$, presenta un cariotipo compuesto por $8 m+12 \mathrm{sm}$ (Fig. 1F), en el brazo largo del par 10sm se observó un satélite punctiforme. El tamaño de sus cromosomas varía de 1,08 a 2,75 $\mu \mathrm{m}$, con una media de $1,81 \mu \mathrm{m}$.

En la meiosis, todas las especies estudiadas presentaron comportamiento regular ya que se observaron exclusivamente bivalentes (ver Fig. 3).

\section{Discusión}

Vernonia chamaedrys habita en campos altos del sur de Brasil, Paraguay, Uruguay y el nor- deste de la Argentina donde forma poblaciones notablemente extensas. El número cromosómico observado en esta entidad $(2 n=34)$ coincide con el único recuento anterior efectuado en ejemplares del sur de Brasil (Stutts, 1988).

El presente estudio en $V$. polyphylla $(2 \mathrm{n}=2 \mathrm{x}=34=20 \mathrm{~m}+14 \mathrm{sm})$ difiere de uno anterior realizado con ejemplares de Rio Grande do Sul (Brasil) que cita $2 \mathrm{n}=64=42 \mathrm{~m}+18 \mathrm{sm}+4 \mathrm{st}$ (Ruas et al., 1991).

La subsect. Remotiflorae (Cabrera, 1944) reúne a especies herbáceas y arbustivas con capítulos sésiles dispuestos en cincinos y brácteas de la inflorescencia foliáceas, bien desarrolladas. Dentro de esta subsección, $V$. niederleinii, $V$. teyucuarensis y $V$. glabrata Less. son especies muy afines entre sí que se diferencian so:amente por la forma de las hojas y de los filarios (Cabrera, 1987). Desde el punto de vista citológico las tres entidades se distinguen claramente. $V$. niederleinii tiene $2 \mathrm{n}=90$ y es decaploide con $x=9$, mientras que $V$. teyucuarensis presenta $2 \mathrm{n}=162$ y sería decaoctoploide con $x=9$. El único estudio realizado en $V$. glabrata reporta $n=17$, ca. $17+B s, 51$ (51II), 51 (48II+6I), 52, ca. 67 o 68 (Jones, 1979).

Los niveles de ploidía observados en $V$. nuederleinii (decaploide) y $V$. teyucuarensis (decaoctoploide) son aparentemente los más altos registrados en el género. La poliploidía es un fenómeno bastante común en especies de Vernonia del nuevo mundo (Jones, 1979). Alrededor del $25 \%$ de las entidades americanas analizadas son poliploides, en su mayoría tetraploides u octoploides. 


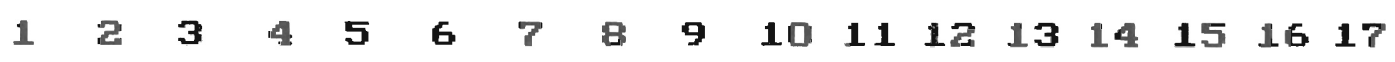

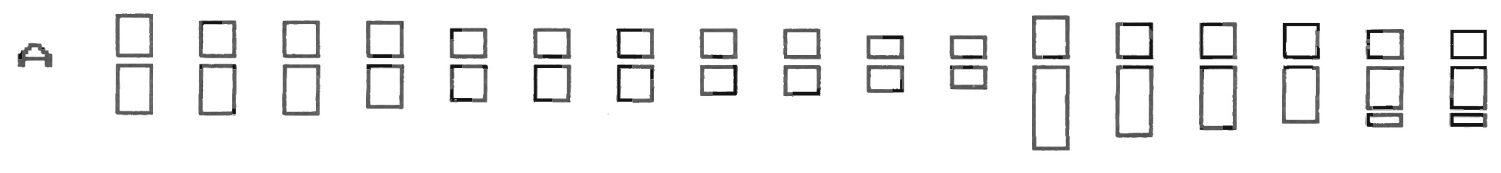

$\mathrm{m}$

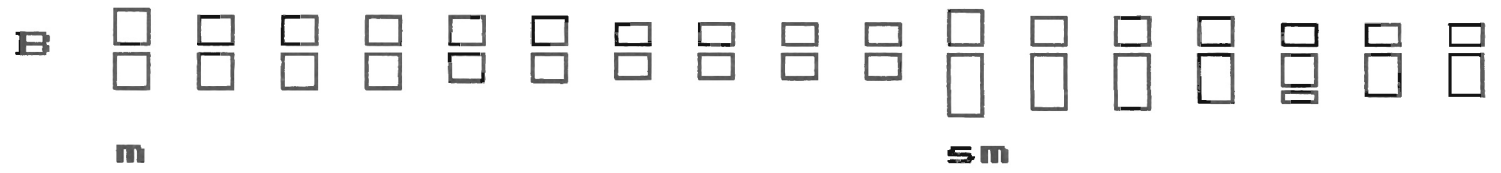

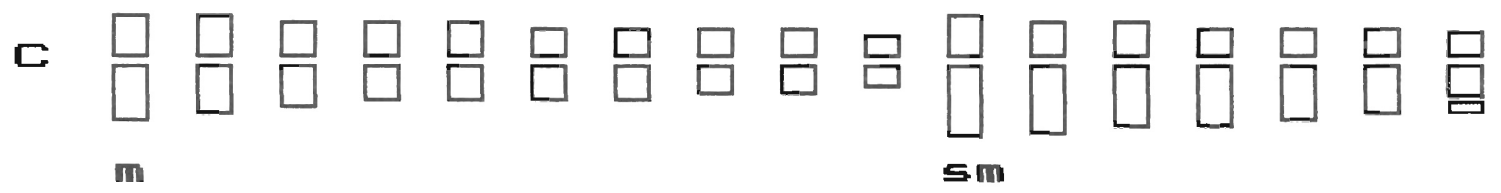

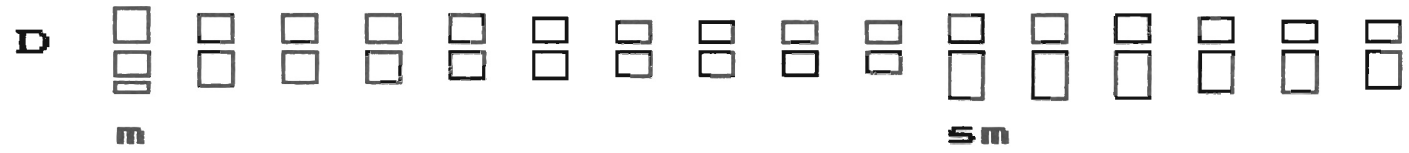

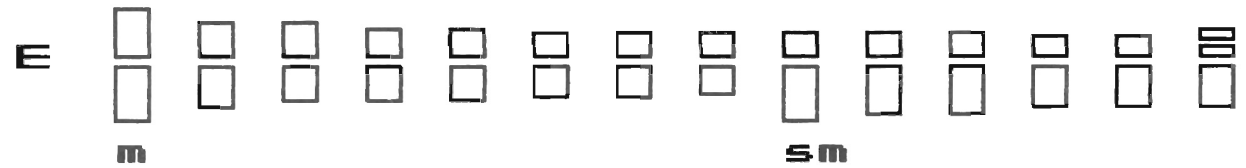

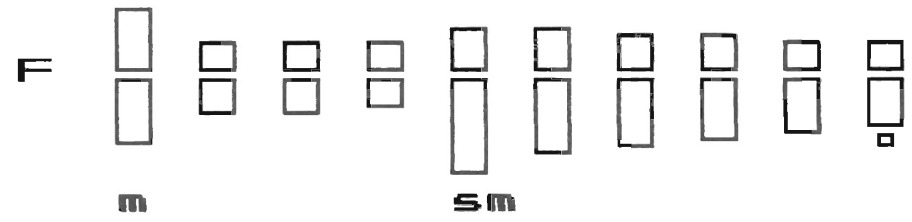

Fig. 1. idiogramas de ias seis especies de Vernonia: A, V. chamaedrys, $22 \mathrm{~m}+12 \mathrm{sm}$; $\mathrm{B}, \mathrm{V}$. loretensis, 20m $+14 \mathrm{sm}$; C, V. polyphylla, 20m $+14 \mathrm{sm} ; \mathrm{D}$, V. rubricaulis, $20 \mathrm{~m}+12 \mathrm{sm} ; \mathrm{E}$, V. remotiflora, $16 \mathrm{~m}+12 \mathrm{sm} ; \mathrm{F}$, V. lepidifera, $8 \mathrm{~m}+12 \mathrm{sm}$. La escala representa $2,5 \mu \mathrm{m}$.

Los resultados observados en $V$. rubricaulis $(\mathrm{n}=16,2 \mathrm{n}=32)$ no coinciden con los de Jones (1970), quién describe $n=33$ en material de nuestro país pero sin citar testigos. Si bien Ruas et al. (1991) mencionan $x=8$ para Vernonia, lo único que sugeriría la existencia de este número básico es el reporte de $\mathrm{n}=$ ca. 8 en $V$. pacchensis Benth. var. tambillensis Hieron.
(Turner et al., 1967). Hasta ei presente no se ha observado $2 n=16$ en nungún taxón de Vernonia. Si dicho recuento fuera correcto se podría considerar tetraploide con $\mathrm{x}=8$ a $\mathrm{V}$. rubricaulis; de lo contrario esta especie sería diploide con $x=16$. Deberían realizarse nuevos estudios en $V$. pacchensis y especies relacionadas para comprobar la validez de $\mathrm{x}=8$. 

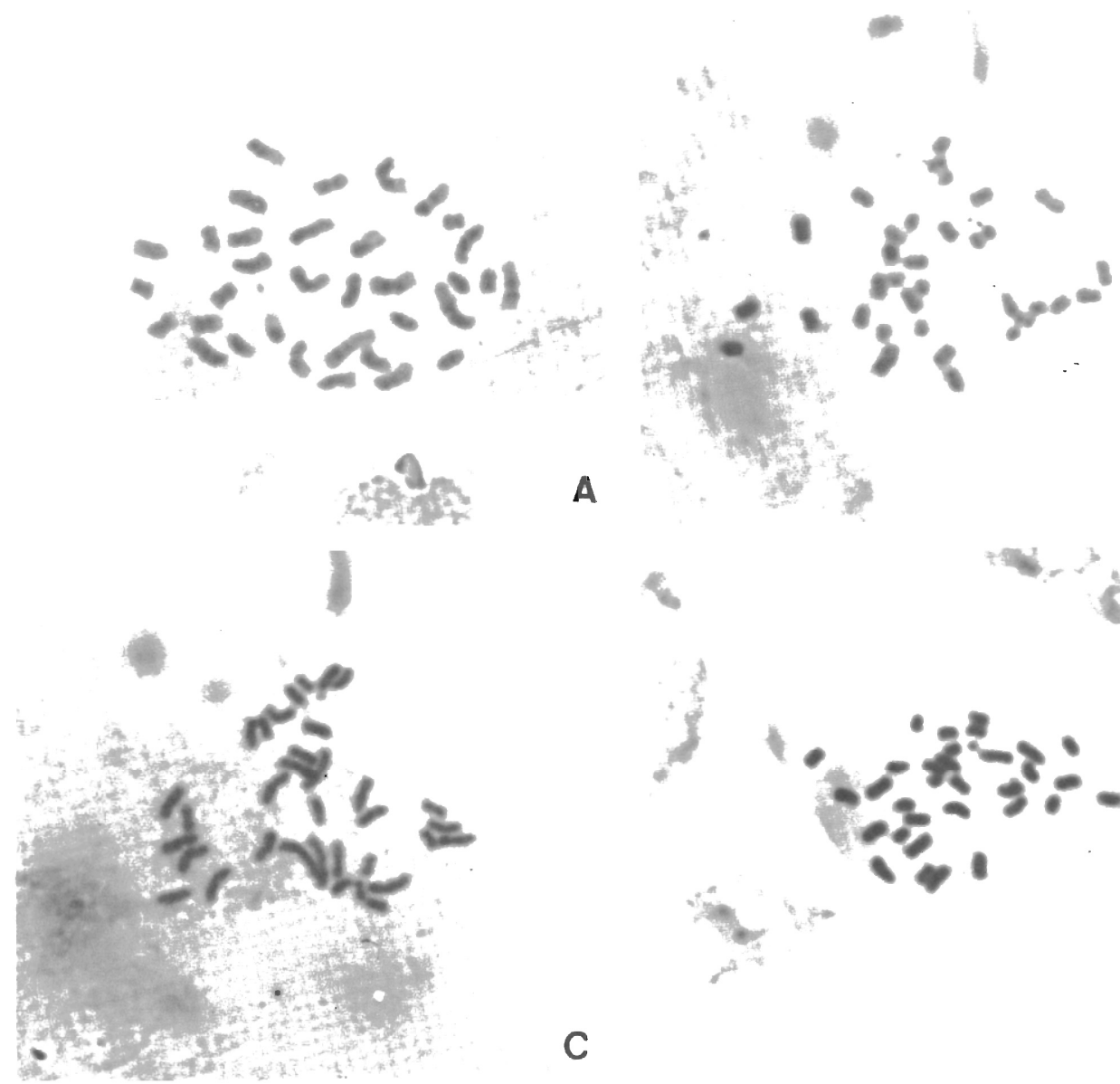

A

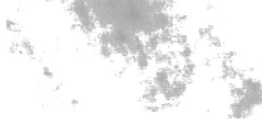

B

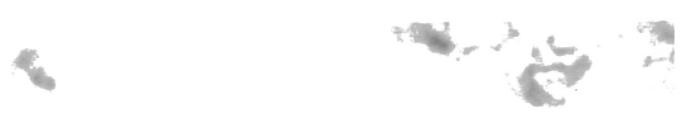

C
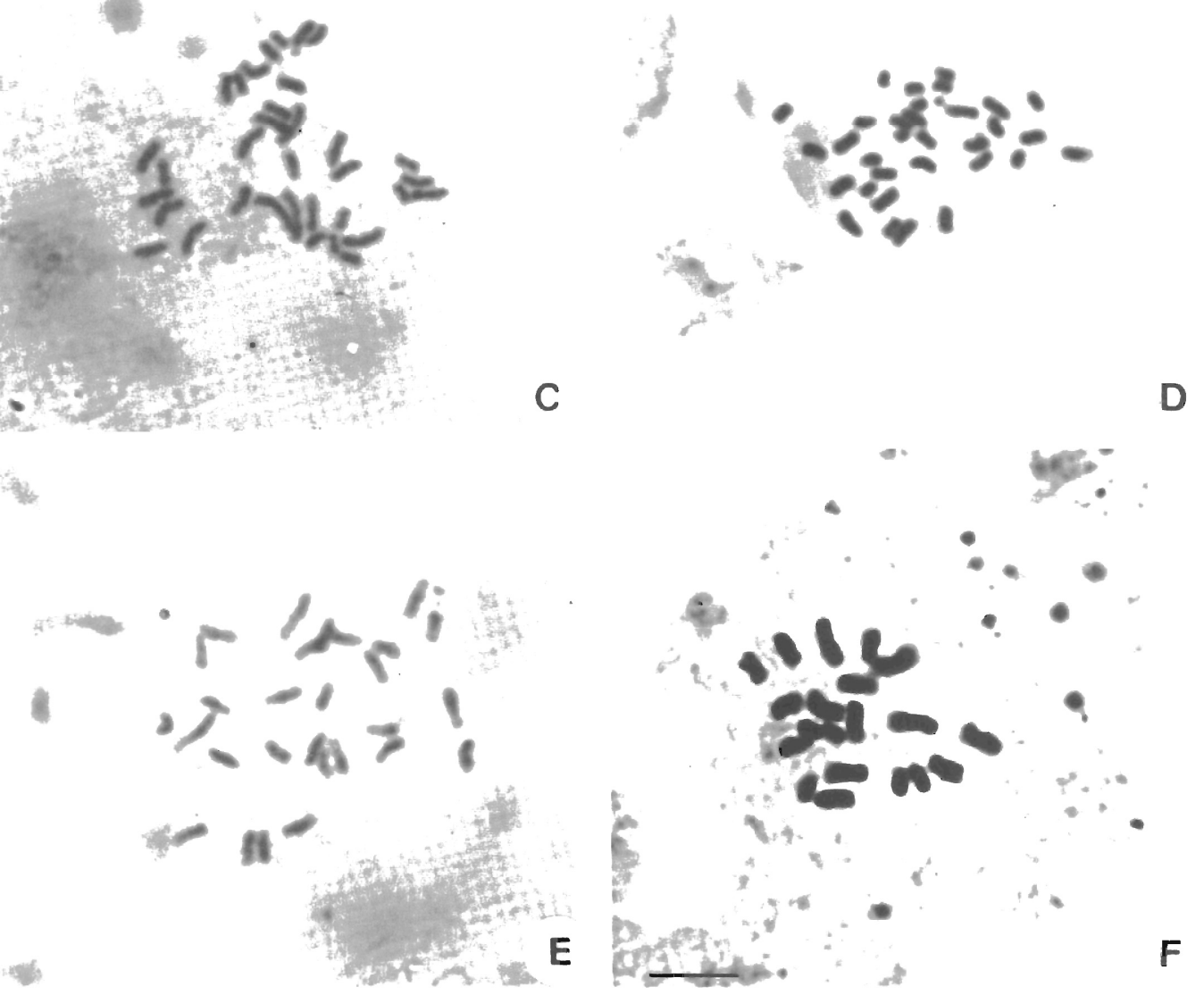

Fig. 2. Mitosis: $A, V$. chamaedrys, prometafase, $2 n=34 ; B, V$. loretensis, metafase, $2 n=34 ; C, V$, polyphylla, metafase, $2 \mathrm{n}=34$; $D, V$. rubricaulis, metafase, $2 n=32 ; E, V$. remotiflora, prometafase, $2 n=28 ; F, V$ lepidifera, metafase, $2 n=20$. La escala representa $5 \mu \mathrm{m}$. 

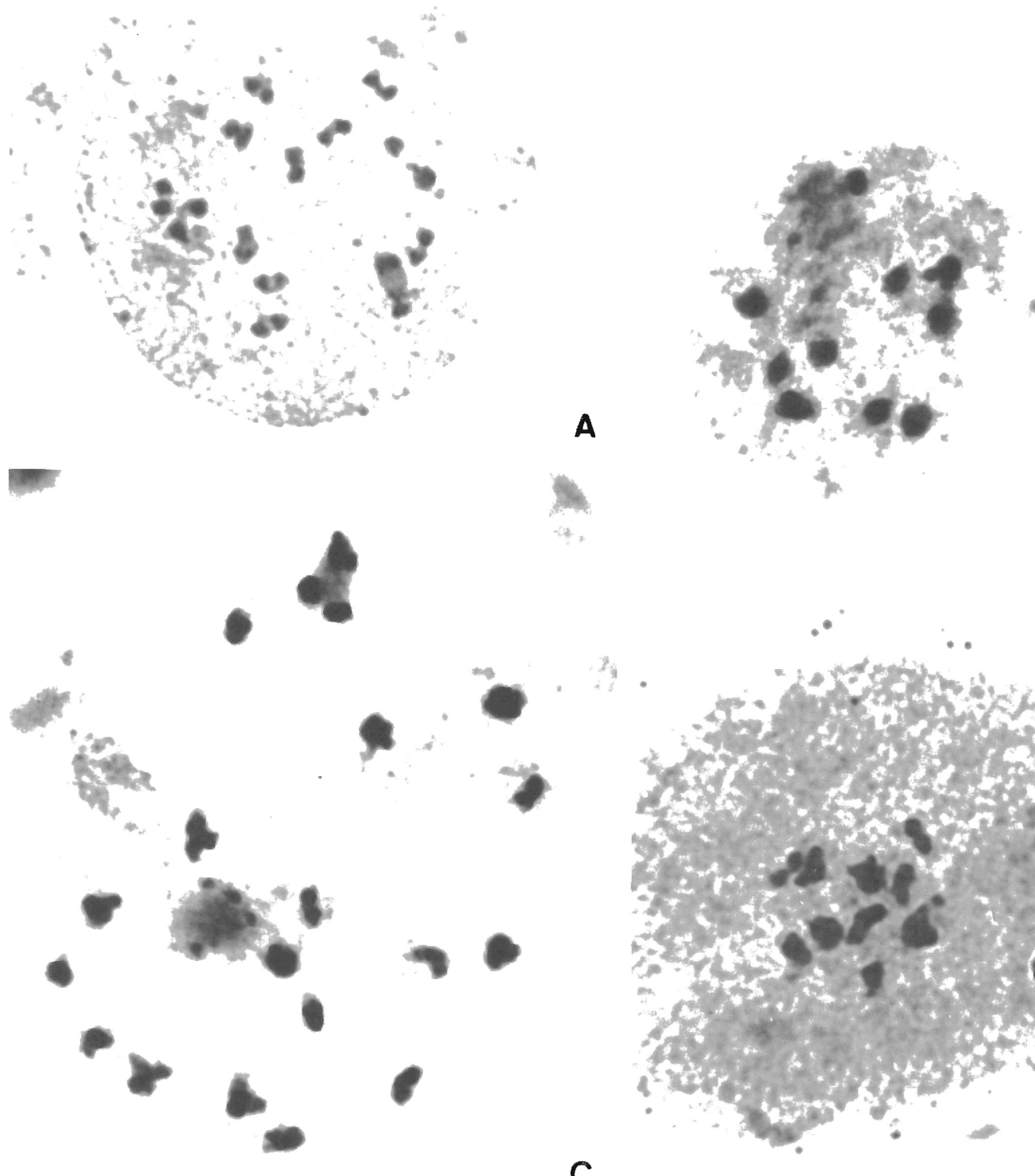

B

C

D

Fig. 3. Meiosis: A, V remotiflora, diacinesis, 14 I; B, V. verbascifolia, diacinesis, 10 II; C, V. platensis, diacinesis, 20 II; D, V. lepidifera, diacinesis, $10 \mathrm{I}$. La escala representa $5 \mu \mathrm{m}$.

Rabakonandrianina \& Carr (1987) han propuesto $\mathrm{x}=7$ para Vernonia sobre la base de un recuento efectuado en $V$. appendiculata Less., una especie endémica de Madagascar. Por lo que $V$. remotiflora, estudiada aquí por primera vez, podría ser considerada un tetraploide con dicho número básico. Teniendo en cuenta que en la meiosis presenta únicamente bivalentes, se trataría de un alotetraploide. Esto confirma- ría la existencia de $x=7$, que constituye el número básico más bajo hallado hasta ahora en Vernonia.

$V$. verbascifolia, por el tipo de inflorescencia que posee, fue incluida en un principio dentro de la subsect. Remotiflorae (Cabrera, 1944). Posteriormente, Jones (1981) la transfirió a la serie Flexuosae (subsect. Flexuosae Cabrera), basándose sobre todo en datos palinológicos y 
bioquímicos. El presente recuento de $n=x=10$ en $V$. verbascifolia apoyaría la posición de Jones (1981), debido a que el número básico $x=10$ es característico de especies de la subsect. Flexuosae. Dentro de esta subsección, $V$. verbascifolia estaría relacionada con $V$. platensis (Jones, 1981), de la que se diferencia por la disposición de las brácteas de la inflorescencia, el tamaño de los capítulos y la longitud de los filarios.

$V$. lepidifera y $V$. platensis pertenecen a la subsect. Flexuosae, que agrupa a hierbas perennes provistas de xilopodio, con capítulos sésiles dispuestos en cincinos alargados y brácteas de la inflorescencia reducidas. Ambas especies se separan por el número de flores y por la forma $\mathrm{y}$ disposición de las hojas. El hallazgo de $2 \mathrm{n}=20$ en $V$. lepidifera concuerda con datos citológicos de otras especies de la subsección. Para la mayor parte de ellas se ha citado $x=10$, número básico muy frecuente en las especies de Vernonia del viejo mundo, pero no en las del nuevo mundo, donde $\mathrm{x}=17$ constituye el número básico modal.

En $V$. platensis $(2 \mathrm{n}=20)$ trabajos anteriores citan $n=10$ (Hunter, 1964), $\mathrm{n}=20$ (Núñez en Cabrera, 1944) y $\mathrm{n}=68$ (Jones, 1982). Galiano y Hunziker (1987) observan $n=10$ y $n=20$ en individuos de diferentes poblaciones, por lo que plantean la existencia de dos citotipos, uno diploide y otro tetraploide con $x=10$. El material analizado en el presente trabajo correspondería entonces al citotipo tetraploide de $V$. platensis.

Los números básicos más frecuentes en Vernonia son $\mathrm{x}=9, \mathrm{x}=10 \mathrm{y} \mathrm{x}=17$, pero no serían los únicos del género como sugiere Jones (1979). Además de éstos y de los números bás:cos mencionados anteriormente, se han citado especies con $x=11$ (Gill \& Omoigui, 1987), $x=12$ (Strother, 1983), $\mathrm{x}=15$ (Ruas et al., 1991) y $\mathrm{x}=19$ (Jones, 1973; Turner, 1981; Sundberg et al., 1986). El número básico considerado ancestral para Vernonia es $\mathrm{x}=9$ (Jones, 1977). Este podría haber derivado de un número inicial $x=4$ o $x=5$, propuestos por Turner (1977) como posibles números básicos de la familia. Es probable entonces, que $x=7$ se haya originado a partir de $\mathrm{x}=9$ por aneuploidía.

Aún resta mucho por conocer sobre la citología de este género, pero es evidente que la aneuploidía y la poliploidía han tenido un rol importante en la especiación de Vernonia.

\section{Agradecimientos}

Al Ing. Agr. Aveliano Fernández por su apoyo desinteresado y sugerencias, a la Dra. Cármen L. Cristóbal por la lectura crítica del manuscrito y la verificación de algunas determinaciones.

\section{Bibliografía}

BERNARDELLO, L. M., 1986. Números cromosómicos en Asteraceae de Córdoba. Darwiniana 27: 169-178.

CABRERA, A. L., 1944. Vernonieas Argentinas (Compositae). Darwiniana 6 (3): 265-379.

1987. Una nueva especie de Vernonia (Compusitae) de Misiones, República Argentina. Darwiniana 28: 187189

COLEMAN, J. R., 1968. Chromosome numbers in some brazilian Compositae. Rhodora 70: 228-240.

COVAS, G. \& J. H. HUINZIKER, 1954. Estudios cariológicos en Antófitas. Revista Invest. Agric. 8: 249-253.

FERNANDEZ, A., 1973. El ácido láctico como fijador cromosómico. Bol. Soc. Argent. Bot. 15: 287-290.

GALIANO, N. G. \& J. H. HUNZIKER, 1987. Estudios cariológicos en Compositae. IV.Vernonieae y Eı:patorieae Darwiniana 28: 1-8.

GILL, L. S. \& I. D. OMOIGUT, 1987. The incidence of polyploidy in family Asteraceae in southern Nigeria. Rev. Cytol. Biol. Vég., Bot. 10: 177-184.

HUNTER, G. E., 1964. Chromosome numbers in Verionia: Section Lepidaploa Subsection Paniculatae verae. Southw. Naturalist 9: 239-244.

HUNZIKER, j. H., A. ESCOBAR, C. C. XIFREDA \& j. C. GAMERRO, 1990. Estudios cariológicos en Compositae. VI. Darwiniana 30: 115-12i.

JONES, S. B., 1970. Chromosome numbers in Compositae. Bull. Torrey Bot. Club 97: 168-174.

- 1973. Revision of Vernonia sect. Eremosis (Compositae) in North America. Brittonia 25: 86-115.

- 1974. Vernonieae (Compositae) chromoscme numbers. Bull. Torrey Bot. Club 101. 3i-34.

— 1977. Vernonieae - systematic review. In: Heywood, V. H., J. B. Harborne \& B. L. Turner (Eds.). The Biology and Chemistry of the Compositae. $Y$. Academic Press, London.

- 1979. Chromusome numbers of Vernonieae (Compositae). Bull. Torrey Bol. Club 106: 79-84.

-1981. Revision of Vernonia series Flexuosae (Compositae: Vernonieae). Brittonia 33: 214-224.

- 1982. En IOPB chromosome numbers reports LXXIV. Taxon 31: 126-127.

LEVAN, A., K. FREDGA \& A. A. SANDBERG, 1964. Nomenclature for centromeric position on chromosomes. Hereditas 52: 201-220.

RABAKONANDRIANINA, E. \& G. D. CARR, 1987. Chromosome numbers of Madagascar plants. Ann 
Missouri Bot. Gard. 74: 123-125.

RUAS, ?. M., C. F. RUAS, A. O. S. VIEIRA, N. I. MATZENBACHER \& N. S. MARTINS, 1991. Sytogenetics of genus Vernonia Schreber (Compositae). Cytologia 56: 239-247.

STROTHER, !. L., 1983. More chromosome studies in Compositae. Amer. J. Bot. 70: 1217-1224.

STUTTS, J. G., 1988. Taxonomic revision ofVernonia Subsect. Chamaedrys (Compositae: Vernonieae). Rhodora 90: 3799.

SUNDBERG, S., C. '. SOWAN \& B. L. TURNER, 1986. Chromusome counts in Latin American Compositae.
Amer, j. Bot. 73: 33-38.

TURNER, B. L., 1977. Summary of the biology of the Compositae. In: Heywood, V. H., J. B. Harborne \& B. L: Turner (Eds.). The Biology and Chemistry of the Compositae. II. Academic Press, London.

1981. New species and combinations in Vernonia sections Leiboldia and Lepidonia (Asteraceae), with a revisional conspectus of the groups. Brittonia 33: 401 412.

TURNER, B. L., A. M. POWELL \&J.CUATRECASAS, 1967. Chromosome numbers in Compositae. II. Peruvian species. Ann. Missouri Bot. Gard. 54: 172-177. 